\title{
CHARACTERIZATION OF SILK FIBROIN/HYALURONIC ACID POLYELECTROLYTE COMPLEX (PEC) FILMS
}

\author{
Ö. Malay, D. Yalçın, A. Batıgün and O. Bayraktar* \\ Biochemical Engineering Research Laboratory (BERL), Department of Chemical Engineering, Izmir Institute of Technology \\ Gülbahçe Köyü, 35430, Urla-Izmir, Turkey
}

\begin{abstract}
This study aimed the characterization of the films casted from the aqueous mixtures of the $\mathrm{pH}$ induced complexes between silk fibroin (SF) and hyaluronic acid (HA). The insoluble and transparent films were subjected to scanning electron microscopy (SEM) analyses to show the morphological changes. Thermal analysis of complex films was determined by a differential scanning calorimeter (DSC). The changes in the crystalline state were monitored by X-ray diffractometer (XRD) and Fourier transform infrared spectroscopy (FTIR). It was shown that the complexation between HA and SF was dominantly induced by pH. It was shown that the complex films comprised mixtures of crystalline and non-crystalline regions.
\end{abstract}

Keywords: DSC, FTIR, hyaluronic acid, polyelectrolyte complex, silk fibroin, XRD

\section{Introduction}

The phenomena of interpolymer interactions have been the focus of intensive fundamental and applied research on polyelectrolyte complexes (PECs). The PECs prepared from natural polymers such as proteins and polysaccharides have the additional advantage of being non-toxic and bioabsorbable [1]. Proteinpolyelectrolyte interactions have been concerned widely with biotechnological and biomedical applications such as protein purification, enzyme immobilization, immunosensing and bioactive sensors [2, 3]. Several globular proteins and polyelectrolytes (natural or synthetic) have been matched for various techniques and objectives of investigation. Table 1 represents some examples of researches on the formation of protein/ polysaccharide systems in literature.

Protein-polyelectrolyte interactions often leading to coacervation and resulting in biopolymer complexes arises mainly from electrostatic interactions [16]. This complexation is dependent on the ionization degree of these macromolecules and thus the $\mathrm{pH}$. The aqueous complexation mixtures obtained under specific conditions can be processed into several forms such as films, gels, sponges, etc. The recent trend is to replace the natural biopolymers used with the new ones to allow precise control over resultant material properties [17]. Several researches have been concentrated on the PECs that occur in protein-polyelectrolyte mixtures. The formation of silk fibroin and hyaluronic acid complex coacervates has been reported by our group earlier [17]. Fibroin, a fibrous silk protein, is produced by silkworm Bombyx mori, is an excellent film former. This protein has been found to be a good starting material for preparation of fibroin-based materials such as gel, powder or film and it has potential uses in contact lenses, artificial corneas, burn wound dressings, artificial lungs and drug delivery devices [18]. On the other hand, HA is a natural anionic polysaccharide that has been used in the formation of PECs. It is thought as an attractive building block for the production of novel biomaterials with potential applications in drug delivery [19-22] and tissue engineering [23]. The specific objective of this work was to characterize the films produced by complex formation between SF and HA.

\section{Experimental}

\section{Materials}

Silk fibroin (SF) was obtained in reeled form from Bursa Institute for Silkworm Research (Bursa/Turkey). Hyaluronic acid (HA) sodium salt ( $M_{\mathrm{W}}: 1600000 \mathrm{Da}$, form Streptococcus equi) was provided by Fluka-BioChemica (Buchs, Switzerland) in powder form. Di-sodium hydrogen phosphate, phosphate dihydrogen phosphate (acid and base components for phosphate buffer), ethanol (absolute GR for analysis) and sulfuric acid $(98+\%)$ were from Merck (Darmstadt, Germany). Calcium chloride-2-hydrate and sodium chloride were supplied from Riedel-de Haën (Seelze, Germany), sodium carbonate $(99.5+\%)$ was from Aldrich-Chemie (Steinheim, Germany) and timolol

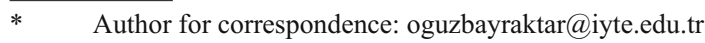


Table 1 Some examples of protein/polysaccharide systems studied in literature

\begin{tabular}{|c|c|c|}
\hline Systems & Experimental techniques & References \\
\hline Gelatin/gum acacia & light microscopy, viscosity, turbidity, phase diagrams & [4] \\
\hline Gelatin/gum acacia & microelectrophoretic mobility & [5] \\
\hline Gelatin/gelatin & microelectrophoretic mobility, preparation of gelatin microcapsules & [6] \\
\hline Gelatin/gelatin & $\begin{array}{l}\text { microelectrophoretic mobility, coacervate yield determination, photon } \\
\text { correlation spectroscopy }\end{array}$ & [7] \\
\hline $\begin{array}{l}\text { Gelatin/gum acacia; bovine serum } \\
\text { albumin/gum acacia }\end{array}$ & microelectrophoretic mobility, dry coacervate yield determination & {$[8]$} \\
\hline Bovine serum albumin/gum acacia & microelectrophoretic mobility, dry coacervate yield determination & [9] \\
\hline $\begin{array}{l}\text { Gelatin/gum acacia; bovine serum } \\
\text { albumin/gum acacia }\end{array}$ & microcapsule production, particle size stability, scanning electron microscopy & [10] \\
\hline Faba bean legumin/chitosan & $\begin{array}{l}\text { ultraviolet spectroscopy, viscometry, calorimetry, turbidimetric titration, } \\
\text { surface tension, emulsion stability }\end{array}$ & [11] \\
\hline$\beta$-Lactoglobulin/gum acacia & $\begin{array}{l}\text { confocal laser microscopy, small angle static light scattering (SASLS), time } \\
\text { resolved sasls, turbidity }\end{array}$ & [12] \\
\hline Casein micelles/pectin & dynamic light scattering, adsorption measurements, renneting experiments & [13] \\
\hline $\begin{array}{l}\beta \text {-Lactoglobulin/pectin (low- and } \\
\text { high-methylated) }\end{array}$ & $\begin{array}{l}\text { potentiometric titrations, determination of the quantity of } \beta-\lg \text { complexed to } \\
\text { pectin (ultrafiltration) }\end{array}$ & [14] \\
\hline $\begin{array}{l}\beta \text {-Lactoglobulin/pectin (low- and } \\
\text { high-methylated) }\end{array}$ & $\begin{array}{l}\text { isothermal titration calorimetry (binding constant, stoichiometry, enthalpy, } \\
\text { entropy), overlapping binding site model }\end{array}$ & [15] \\
\hline Whey protein/gum arabic & small angle $\mathrm{X}$-ray scattering, turbidimetric titration & [16] \\
\hline Silk fibroin/hyaluronic acid & viscometry, turbidimetric titration, electrophoretic mobility & [17] \\
\hline
\end{tabular}

maleate salt $\left(M_{\mathrm{W}}=432.5\right)$ was from Sigma (St. Louis, MO, USA). Dialysis tubing ( $M_{\mathrm{W}}$ Cut-off: $12000-14000$ ) was obtained from Sigma (St. Louis, MO, USA) and Medicell (London, UK). Sodium sulfide hydrate was provided by Fluka Chemie (Buchs, Switzerland). Deionized water was used during all experiments.

\section{Preparation of stock solutions}

Silk fibroin solutions were prepared by subsequent processes of degumming and dissolution. During the degumming process, raw silk was kept in 50 times $(\mathrm{v} / \mathrm{w})$ of boiling aqueous $0.05 \% \mathrm{Na}_{2} \mathrm{CO}_{3}$ for $30 \mathrm{~min}$ and this treatment was repeated 3 times. This was followed by washing several times with deionized water and the degummed silk was left drying at room temperature. To obtain aqueous SF solution, $1.2 \mathrm{~g}$ degummed silk was added to 20 times $(v / w)$ of Ajisawa's reagent $\left(\mathrm{CaCl}_{2} /\right.$ ethanol/water, $111 / 92 / 144$ in mass $)$ in a Sholtz bottle with a volume of $250 \mathrm{~mL}$ [24]. The mixture was stirred at $78^{\circ} \mathrm{C}$ to form a clear solution for $2 \mathrm{~h}$. The resulting SF solution was then dialyzed against deionized water for at least 3 days at sub-ambient temperature to remove the neutral salts using a cellulose tube ( $M_{\text {WCO }} 12000-14000$, Sigma). The dialysis was ended as the dialysate tested negative for chloride ion by performing silver chloride precipitation test using $\mathrm{AgNO}_{3}$. The dialyzed fibroin solution is filtered with a Filtrak 389 filter paper (Barenstein, Germany). The pure aqueous fibroin solution with a concentration of $1-2 \%(\mathrm{~m} / \mathrm{v})$ was obtained after filtration. The concentration of the SF solution was controlled using a rotary vacuum evaporator, Heidolph Laborota 4001, run at $30^{\circ} \mathrm{C}$ and $30 \mathrm{rpm}$.

HA was provided in powder form and it was soluble in water or any buffer solutions considered. However, HA particles were prone to coagulation during dissolution; therefore HA solution was stirred overnight to ensure complete solubilization.

\section{Preparation of SF-HA films}

Regenerated silk fibroin (RSF) solution adjusted to desire concentration and sodium hyaluronate dissolved in water are mixed at a preset ratio. The $\mathrm{pH}$ of the solution was concurrently monitored and the $\mathrm{pH}$ was adjusted to $\mathrm{pH}$ value between the $\mathrm{pK}_{\mathrm{a}}$ of $\mathrm{HA}$ and IEP of SF. The mixture was stirred for $4 \mathrm{~h}$ and casted on polyethylene Petri-dishes (diameter $=5 \mathrm{~cm}$ ) at room temperature. The casted films were dried at $20^{\circ} \mathrm{C}$ and at $80 \% \mathrm{RH}$ for the first $2 \mathrm{~h}, 65 \% \mathrm{RH}$ for the following 2 days in an environmental chamber (Angelantoni Industrie, Italy). The preparation conditions and type of the complexes were given in Table 2 .

The dried films were stored in a desiccator at $10^{\circ} \mathrm{C}$ until used to avoid contamination. The solubility of the films was controlled by immersing in deionized water and buffer solutions. 
Table 2 Detailed description of the preparation conditions of the complexes

\begin{tabular}{ccccc}
\hline Film label & $\mathrm{pH}$ & $\mathrm{r}(\mathrm{SF}:$ HA mass ratio) & Drying condition & Description \\
\hline F1 & 3.2 & - & $45^{\circ} \mathrm{C}, 65 \% \mathrm{RH}$ & pure SF film \\
F2 & 5.5 & - & $20^{\circ} \mathrm{C}, 65 \% \mathrm{RH}$ & pure SF film \\
F3 & 5.5 & 20 & $20^{\circ} \mathrm{C}, 65 \% \mathrm{RH}$ & SF-HA film \\
F4 & 3.2 & 20 & $20^{\circ} \mathrm{C}, 65 \% \mathrm{RH}$ & SF-HA film \\
F5 & 3.2 & 30 & $20^{\circ} \mathrm{C}, 65 \% \mathrm{RH}$ & SF-HA film \\
F6 & 3.2 & 30 & $20^{\circ} \mathrm{C}, 65 \% \mathrm{RH}$ & SF-HA coacervate particles \\
F7 & 3.2 & 20 & room conditions & SF-HA film \\
F8 & 3.2 & - & $20^{\circ} \mathrm{C}, 65 \% \mathrm{RH}$ & pure HA film \\
\hline
\end{tabular}

\section{Instrumental methods}

Thermal analysis of SF-HA coacervates and complex films were determined by a differential scanning calorimeter (DSC) (Schimadzu DSC-50) in the $25-450^{\circ} \mathrm{C}$ temperature range at a scan rate of $10^{\circ} \mathrm{C} \mathrm{min}^{-1}$ using stainless-steel pans under nitrogen. Scanning electron microscopy (SEM) (Philips XL 30S FEG) analyses were performed to show the morphological changes in the membranes. The films were coated with gold-palladium by Polaron SC 7610 Sputter Coater prior to imaging.

The changes in the crystalline state were monitored by X-ray diffractometer (XRD) (Philips X'pert Pro) with $\mathrm{CuK}_{\alpha}$ radiation for $2 \theta$ from 7 to $70^{\circ}$. Fourier transform infrared spectroscopy (FTIR) analysis was carried out in the spectral region of $700-4000 \mathrm{~cm}^{-1}$ using a FTIR spectrophotometer (Digilab FTS 3000 Mx) equipped with ATR diamond cell accessory.

\section{Results and discussion}

Formation and characterization of SF-HA complex films

The complex films dried at the proposed $\mathrm{pH}$ window (pH 2.5-3.5) under controlled temperature and relative humidity resulted in homogeneous transparent films. Mechanical properties of the materials are of primary importance for determining the performance in case of varying stress conditions. The pure SF film displayed the typical behavior of brittle materials, as reported [25]. On the other hand, SF-HA complexes were effective in inducing only a slight improvement on the mechanical properties of SF films. As HA content increased, more flexible films at dry state were obtained. The films were much more flexible before washed to remove excess of HA.

\section{Morphological examination of the SF-HA complex films}

Milky suspension of fine coacervates formed by the complexation of SF and HA casted and dried at $20^{\circ}$ and $65 \% \mathrm{RH}$. The resultant film was examined by

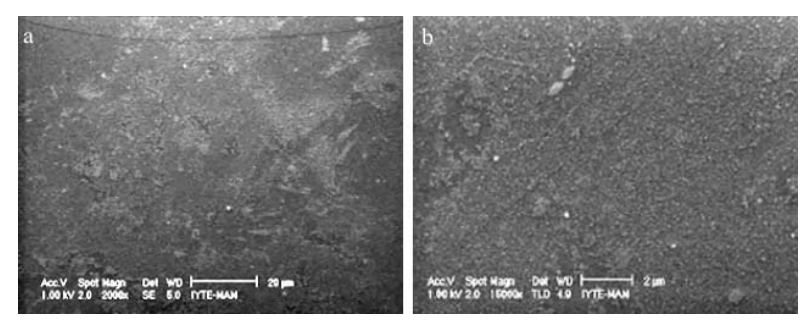

Fig. 1 SEM photographs of the SF-HA complex film with a magnification of $a-2000 \times$ and $b-15000 \times$

SEM. Insoluble SF-HA complex films (F4 and F5) had smooth surfaces with finely distributed roundish particles as shown in Fig. 1. In other words, the films displayed so-called sea-island morphology, which was also met in the SEM photographs of chitosan-alginate polyelectrolyte complexes [26].

\section{Thermal analysis}

DSC is one of the techniques that has been applied to study the molecular conformation of silk-based materials and hence their physical and structural characteristics. Figure 2 below represented the DSC curves of the films prepared at differing conditions. The secondary structure of $B$. mori silk fibroin (SF) consists of the major conformation including random coils (silk I) and $\beta$-sheet (silk II). The following evaluation of the molecular conformation of SF did not differentiate between random coils and silk I structures. It was claimed that distinction between the silk I and random coil was impossible even from IR spectra [27]. For this reason, both conformations were shown as silk I.

The pure SF films, F1 and F2, were both prepared at $\mathrm{pH} 5.5$, but dried at 45 and $20^{\circ} \mathrm{C}$, respectively. Due to heat treatment, F1 was water insoluble and exhibited a strong endothermic peak at $305^{\circ} \mathrm{C}$, which was attributed to thermal degradation of SF film with silk II conformation. On the contrary, F2 film, which was water soluble, showed an exothermic peak around $225^{\circ} \mathrm{C}$ due to crystallization of the amorphous fibroin 


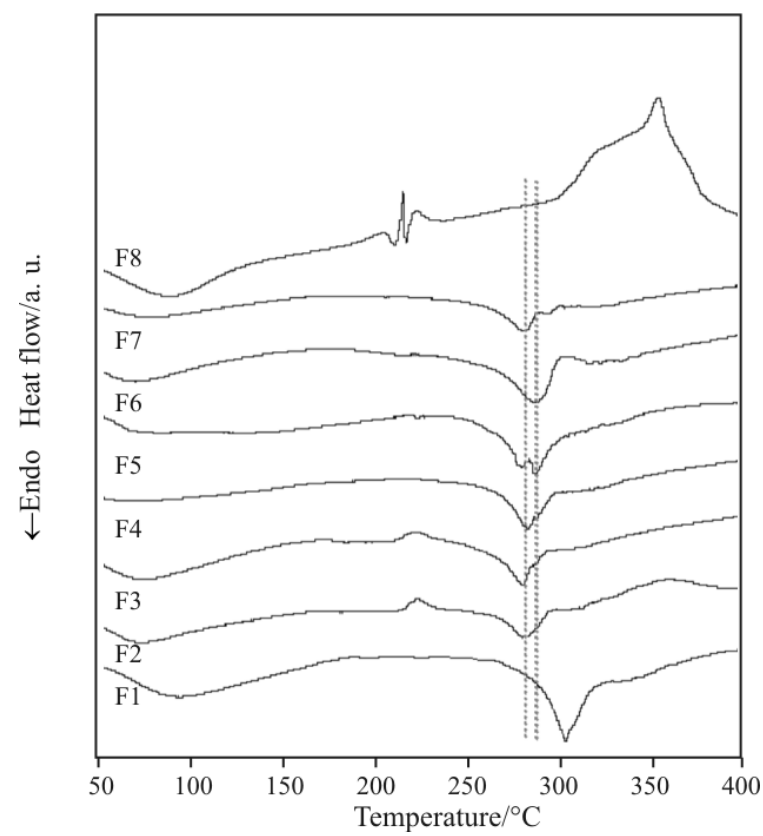

Fig. 2 DSC curves of the films. SF film-II (F1), SF film-I (F2), SF-HA blend (SF:HA=20) (F3), SF-HA complex film (SF:HA=20) (F4), SF-HA complex film SF:HA=30) (F5), phase-separated coacervate network (F6), coacervate film (F7), HA film (F8)

from random coil (silk I) to $\beta$-crystals (silk II). The peak of thermal decomposition of F1 shifted to a lower temperature $\left(285^{\circ} \mathrm{C}\right.$ as shown by the dashed line a) with less intensity, by the lower degree of molecular orientation when compared to DSC trace of the F2 with $\beta$-sheet (silk II) conformation. The peak positions were consistent with reported DSC traces of the silk I and silk II type fibroin [25, 28-30]. F3 represented the DSC trace of the SF-HA physical mixture prepared at $\mathrm{pH} 5.5$ with a SF:HA mass ratio of 20 , which corresponded to 1:1 pairing of the charged groups on the biopolymers. During the preparation of this mixture, no turbidity was seen as naked-eye observation. F3 exhibited a very similar curve to that of F2 with silk I structure. An exotherm was seen at $225^{\circ} \mathrm{C}$ with a lower intensity and a narrower but higher intensity endotherm at $285^{\circ} \mathrm{C}$. The appearance of the endotherm also showed that SF/HA blending did not introduce crystallization. No effect of HA was observed in this curve or either in other curves including HA, of which the two characteristic exothermic peaks was shown with F8 on DSC trace [31]. This was attributed to partition of HA in the mixtures in very small mass amounts. The only difference between the traces of the physical mixture of SF-HA and pure SF film with silk I structure was the appearance of a shoulder around $290^{\circ} \mathrm{C}$ (shown by dashed line b). This was assigned to the formation of soluble interpolymer complexes above the isoelectric point of SF due to local positive charge patches. This was consistent with the obtained $\mathrm{pH}$-window for the formation of soluble complexes in the turbidimetric analysis [17]. On the other hand, preparation of SF-HA complex film at $\mathrm{pH} 3.2$ at the same mass ratio introduced the formation of silk II structure to F4. As soon as two materials were mixed, turbidity (white-milky appearance) was observed during the preparation of the complex mixture at the specified $\mathrm{pH}$. The resultant SF-HA complex film exhibited silk II conformation and thermal degradation endotherm did not shift to a higher temperature as seen in case of F1 with a positive shift of $20^{\circ} \mathrm{C}$. On the other hand, the shoulder seen on F3 around $290^{\circ} \mathrm{C}$ was sharper in case of F4 was attributed to transition of soluble intermolecular complexes to insoluble intrapolymeric complexes, which resulted in insoluble SF-HA complex films. This shoulder turned into an endotherm at the DSC curve F5, which was also prepared at $\mathrm{pH} 3.2$ but at higher SF:HA ratio of 30 . This increase beyond 1:1 stoichiometry was consistent with previous results showing the rearrangement of the charge distribution of the coacervate complexes leading to higher coacervation yields. Therefore, as the SF:HA ratio increases, complexes with higher thermal stability was obtained due to more compact complexation with stronger ionic interactions.

F6 exhibited the DSC trace of the coacervate phase centrifuged, filtered and dried under controlled conditions. Prior to centrifugation, complex mixture was prepared at $\mathrm{pH} 3.2$ with a SF:HA ratio of 30 . This procedure resulted in particles resembling a network like structure. DSC curve of the coacervate phase showed a broader endotherm at $290^{\circ} \mathrm{C}$, which supported the appearance of a new peak at this temperature attributed to SF-HA complexation. The complex mixture prepared under same conditions waited for a gravity-induced phase separation, which was established in $2 \mathrm{~h}$ without centrifugation and left drying at room conditions. DSC curve of the resultant coacervate film, which was partially-soluble, was shown with F7. The endotherm showing the thermal degradation of this film was very similar to that of soluble fibroin film with silk I structure (F2). However, the crystallization exotherm at $225^{\circ} \mathrm{C}$ was not seen and the endotherm showing the coacervate formation just appeared as a plateau. The exothermic peak at $225^{\circ} \mathrm{C}$ given as a marker of silk I conformation disappeared in all films prepared at $\mathrm{pH}$ 3.2. Moreover, the thermal decomposition did not shift to a higher temperature, which shows higher thermal stability gained by silk II conformation, for these cases. This was attributed to $\mathrm{pH}$-induced conformational transition of SF, which probably occurred at a lower yield when compared with the intensity and decomposition temperature shifts between the DSC traces of F1 and F2. These observations revealed that $\mathrm{SF} / \mathrm{HA}$ complex films did not induce a 
transition to $\beta$-sheet conformation and complexation increased the thermal stability by $10^{\circ} \mathrm{C}$ which may be concluded as the result of the specific interactions between SF and HA, whereas SF in the complex films underwent a transition to $\beta$-sheet by some degree.

\section{$X$-ray diffraction analysis}

Figure 3 represented the XRD patterns of F1 (SF film with silk II conformation), F2 (SF film with silk I conformation), F4 (SF-HA complex film, $r=20$ ), F5 (SF-HA complex film, $r=30$ ) and F8 (HA film) as previously exhibited in DSC analysis. In the figure the DSC patterns of the films were placed in the order of increasing crystallinity. SF film with silk I conformation (F2) showed a weak and wide pattern around $2 \theta=20^{\circ}$ with a spacing of $4.8 \AA$ due to non-crystalline form as the typical characteristic diffraction pattern of amorphous silk fibroin [32]. HA-film also showed up a very broad pattern around $2 \theta=35^{\circ}$. On the other hand, F1 showed a typical diffractogram of $\beta$-sheet crystalline structure, which had three diffraction peaks: a major peak at $17.4^{\circ}$ and two minor peaks at 14.3 and $26.3^{\circ}$. The diffractograms of F4 and F5 showed that crystalline and amorphous phases coexisted SF-HA complex films. F4 $(r=20)$ showed lower crystalline peaks at $14.3,16.9$ and $25.5^{\circ}$ and weak and broad peak around $20^{\circ}$ representing noncrystalline structure, whereas F5 $(r=30)$ exhibited a new broad peak at $7.5^{\circ}$, three crystalline peaks at $14.4,17$ and $25.4^{\circ}$ and a stronger and narrower peak at $20^{\circ}$. The peaks representing the crystalline phases had lower intensity with small shifts when compared to that of F1 and the intensity diminishes as SF:HA mass ratio increased. Small shifts and untypical peaks may be caused by the shear forces being effective on casting and drying, giving rise to $\beta$-sheet characteristics. On the other hand, it was reported that the appearance of unknown peaks or spacings may be due to other types of conformations such as helical and $\beta$-turns or distortions by the presence of conformers and/segments in which torsion angles deviate from those of silk I or silk II [33].

These findings denoted that complexation between $\mathrm{SF}$ and HA through ionic interactions reduced the crystallinity, which was induced by the lower $\mathrm{pH}$ values that favored the $\beta$-sheet conformation for SF. Similar behavior was seen for the chitosan-gelatin polyelectrolyte complex [34]. It was reported that the crystalline peaks appeared on the XRD profiles of the complex became weaker with increasing the gelatin content. The weakening of the crystalline peaks induced by increasing the protein content of the complex was attributed to strong interactions between gelatin and chitosan that led their good compatibility.

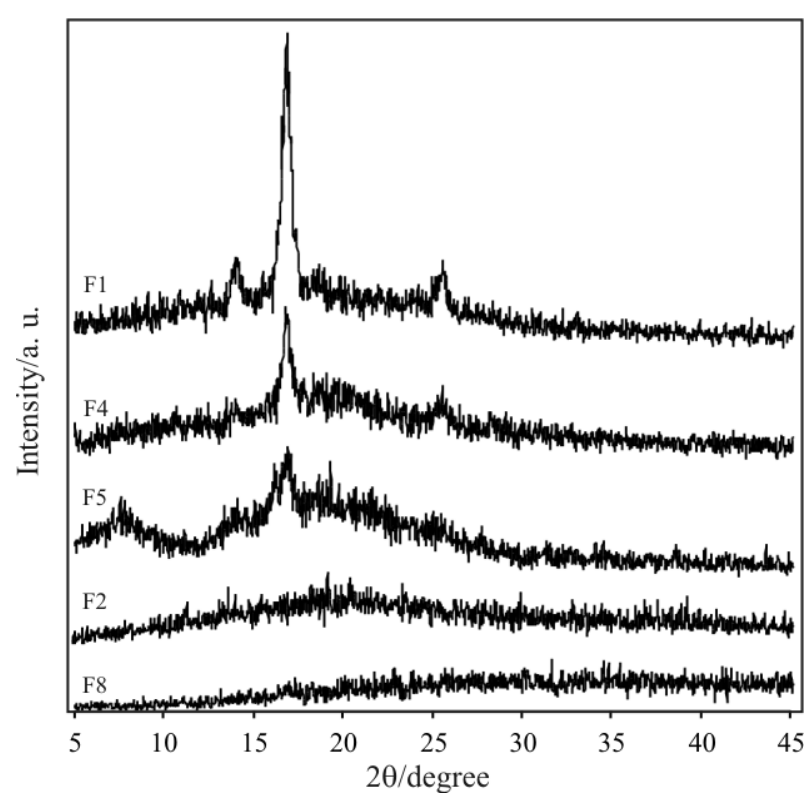

Fig. 3 X-ray diffraction patterns of SF film-II (F1), SF film-I (F2), SF-HA complex film (SF:HA=20) (F4), SF-HA complex film (SF:HA=30) (F5), HA film (F8)

\section{FTIR analysis}

Another instrumental technique, infrared spectroscopy (IR), was employed to determine the molecular conformation and orientation of SF-HA complex films. IR spectrum in the $1800-800 \mathrm{~cm}^{-1}$ range of control can be a fingerprint of prepared films since it contains various absorption bands related to different chemical and structural features of the prepared films. ATR-IR spectra of the SF-HA complex films, Fig. 4c, showed small but still significant changes with respect to the SF control film (Fig. 4a), which was prepared at pH 3.2 to check the conformation transition observed in DSC analysis. SF-HA complex film was also prepared at 3.2, with a SF:HA ratio of 20, simulating the film shown as F4 in the previous analysis. The resultant pure SF film showed slow and partial dissolution in water compared to that SF film prepared at $\mathrm{pH}$ 5.5.

Amide I-IV bands are conformationally sensitive bands for polypeptides and proteins. Their intensity and position of these bands give information about the molecular conformation of the materials examined in IR spectrum. In amide I and II regions of the IR spectra of the films, instead of a single characteristic peak, bands were observed. In literature, amide I (-CO- and $-\mathrm{CN}-$ stretching) appeared to be in the region of $1655-1660 \mathrm{~cm}^{-1}$, amide II (-NH- bending) in the region of $1531-1542 \mathrm{~cm}^{-1}$ and amide III (-CNstretching) at $1230 \mathrm{~cm}^{-1}$ were attributed to silk I conformation. On the other hand, the appearance of amide I in the region of $1620-1630 \mathrm{~cm}^{-1}$, amide II in the region of $1515-1530 \mathrm{~cm}^{-1}$ and amide III at $1240 \mathrm{~cm}^{-1}$ characterized the $\beta$-sheet conformation 


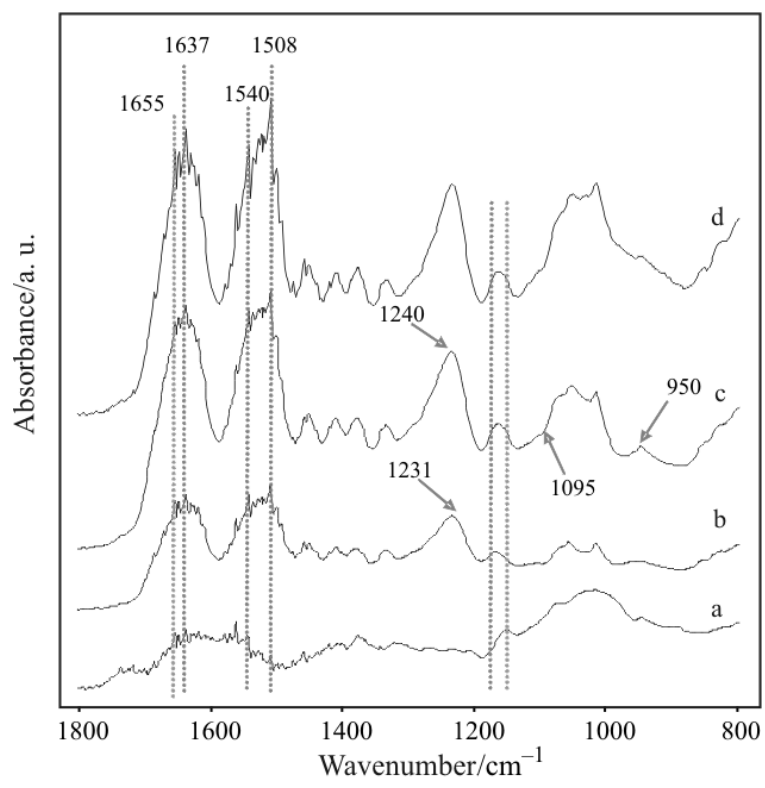

Fig. 4 ATR-IR spectra of the casted films; a - HA, b-SF; c, d-SF-HA complex film

$[26,33,35,36]$. The appearance of strong and broad peak of SF-HA complex film (Fig. 4c) at $1240 \mathrm{~cm}^{-1}$ represented that the dominant conformation was silk I, when compared to the $\beta$-sheet dominant conformation of SF control film (Fig. 4b) which showed $1231 \mathrm{~cm}^{-1}$ absorption band of the silk II conformation. On the contrary, SF-HA complex film, as well as the SF control film, represented absorption bands of 1637 and $1508 \mathrm{~cm}^{-1}$ with accompanying peaks of 1654 and $1540 \mathrm{~cm}^{-1}$. In addition, the appearance of $950 \mathrm{~cm}^{-1}$ (-NH- stretching) absorption band on IR spectra of the complex film favored the existence $\beta$-sheet crystals. Consequently, IR spectra of the films showed that SF-HA complex film, as well as the SF control film, comprised mixtures of silk I $(\alpha)$ and silk II ( $\beta$ ) type conformations and complexation did not introduce a positive effect on crystallization; on the contrary, decreased the crystallinity which may enhance during drying. The $\mathrm{pH}$-induced crystalline structure of SF was disturbed by formation associative compact structures and/or the crystalline phases may be buried into the complexes with respect to higher degree of hydrophobicity due to charge stabilization of the complexes and the $\beta$-sheet conformation.

In addition to all, even though new conspicuous peak did not appear, considerable increase in amide I, amide II, III bands and at $1410 \mathrm{~cm}^{-1}$ corresponding to valency vibration of carboxylate ion and formation of a shoulder at $1095 \mathrm{~cm}^{-1}$ corresponding to $-\mathrm{C}=\mathrm{O}$ stretching for the SF-HA film evidenced the complex formation between the amino groups of SF and carboxyl groups of HA (Figs $4 \mathrm{c}$ and d).

\section{Acknowledgements}

The financial support from Research Fund of İzmir Institute of Technology for this thesis and the general technical support of State Planning Organization on researches in İzmir Institute of Technology are gratefully acknowledged.

\section{References}

1 C. Schmitt, C. Sanchez, S. Desobry-Banon and J. Hardy, Crit. Rev. Food Sci., 38 (1998) 689.

2 C. Schmitt, C. Sanchez, F. Thomas and J. Hardy, Food Hydrocoll., 13 (1999) 483.

3 H. Dautzenberg, Macromol. Symp., 162 (2000) 1.

4 H. R. Kruyt, Colloid Science, Elsevier, Amsterdam 1949, p. 232.

5 D. J. Burgess and J. E. Carless, J. Colloid Interface Sci., 98 (1984) 1

6 D. J. Burgess and J. E. Carless, Int. J. Pharm., 27 (1985) 61.

7 A. Eisenberg and F. R. Bailey, Coulombic Interactions in Macromolecular Systems, ACS Symposium Series 302, American Chemical Society, Washington DC 1986, p. 251.

8 D. J. Burgess, J. Colloid Interface Sci., 140 (1990) 227.

9 D. J. Burgess, K. K. Kwok and P. T. Megremis, J. Pharm. Pharmacol., 43 (1991) 232.

10 P. L. Dubin, Macromolecular Complexes in Chemistry and Biology, Springer Verlag, Berlin 1994, p. 285.

11 E. Dickinson and R. Miller, Food Colloids, Fundamentals of Formulation, Royal Society of Chemistry, Cambridge 2001, p. 332.

12 C. Sanchez, G. Meckloufi, C. Schmitt, D. Renard, P. Robert, C. M. Lehr, A. Lamprecht and J. Hardy, Langmuir, 18 (2002) 10323.

13 R. Tuinier, C. Rolin and C. G. de Kruif, Biomacromolecules, 3 (2002) 632.

14 M. Girard, S. L. Turgeon and S. F. Gauthier, Food Hydrocoll., 16 (2002) 585.

15 M. Girard, S. L. Turgeon and S. F. Gauthier, J. Agr. Food Chem., 51 (2003) 4450.

16 F. Weinbreck, R. H. Tromp and C. G. de Kruif, Biomacromolecules, 5 (2004) 1437.

17 Ö. Malay, O. Bayraktar and A. Batıgün, Int. J. Biol. Macromol., 40 (2007) 38.

18 G. H. Altman, F. Diaz, C. Jacuba, T. Calabro, R. L. Horan, J. Chen, H. Lu, J. Richmound and D. L. Kaplan, Biomaterials, 24 (2003) 401.

19 S. Surini, H. Akiyama, M. Morishita, T. Nagai and K. Takayama, J. Controlled Release, 90 (2003) 291.

20 Y. Luo, K. R. Kirker and G. D. Prestwich, J. Controlled Release, 69 (2000) 169.

21 L. D. Simon, W. N. Charman, S. A. Charman and V. J. Stella, J. Controlled Release, 45 (1997) 273.

22 O. Bayraktar, Ö. Malay, Y. Özgarip and A. Batıün, Eur. J. Pharm. Biopharm., 60 (2005) 373.

23 S. N. Park, J. C. Park, H. O. Kim, M. J. Song and H. Suh, Biomaterials, 23 (2002) 1205.

24 H. Yamada, H. Nakao, Y. Takasu and K. Tsubouchi, Mater. Sci. Eng. C, 14 (2001) 41.

25 Y. Gotoh, M. Tsukada, T. Baba and N. Minoura, Polymer, 38 (1997) 487. 


\section{SILK FIBROIN/HYALURONIC ACID POLYELECTROLYTE COMPLEX FILMS}

26 G. Freddi, M. Tsukada and S. Beretta, J. Appl. Polym. Sci., 71 (1999) 1563.

27 T. Asakura, A. Kuzuhara, R. Tabeta and H. Saito, Macromolecules, 18 (1985) 1841

28 Y. Xu, Y. Zhang, H. Shao and X. Hu, Int. J. Biol. Macromol., 35 (2005) 155.

29 I. C. Um, H. Y. Kweon, Y. H. Park and S. Hudson, Int. J. Biol. Macromol., 29 (2001) 91.

30 E. S. Sashina, G. Janowska, M. Zaborski and A. V. Vnuchkin, J. Therm. Anal. Cal., 89 (2007) 3887.

31 K. Benešová, M. Pekar, L. Lapcík and J. Kucerík, J. Therm. Anal. Cal., 83 (2006) 2341.
32 H. Saitoh, K. Ohshima, K. Tsubouchi, Y. Takasu and H. Yamada, Int. J. Biol. Macromol., 34 (2004) 317.

33 J. Ayutsede, M. Gandhi, S. Sukigara, M. Micklus, H. E. Chen and F. Ko, Polymer, 46 (2005) 1625.

34 Y. J. Yin, K. D. Yao, G. X. Cheng and J. B. Ma, Polym. Int., 48 (1999) 429.

35 H. Wang, W. Li, Y. Lu and Z. Wang, J. Appl. Polym. Sci., 65 (1997) 1445.

36 H. Chen, X. Hu and P. Cebe, J. Therm. Anal. Cal., 93 (2008) 1201.

DOI: $10.1007 / \mathrm{s} 10973-008-9368-5$ 